\title{
Brillouin scattering study on the single-crystal elastic properties of natrolite and analcime zeolites
}

\author{
Carmen Sanchez-Valle and Stanislav V. Sinogeikin \\ Department of Geology, University of Illinois at Urbana-Champaign, 1301, West Green Street, \\ Urbana, Illinois 61801
}

Zoe A. D. Lethbridge and Richard I. Walton

Department of Chemistry, University of Exeter, Stocker Road, Exeter Devon EX4 4QD, United Kingdom

Christopher W. Smith and Kenneth E. Evans

School of Engineering, Computer Sciences and Mathematics, University of Exeter, North Park Road, Exeter EX4 4QF, United Kingdom

\author{
Jay D. Bass \\ Department of Geology, University of Illinois at Urbana-Champaign, 1301, West Green Street, \\ Urbana, Illinois 61801
}

(Received 17 March 2005; accepted 13 July 2005; published online 6 September 2005)

\begin{abstract}
The Brillouin light-scattering technique was used to investigate the single-crystal elastic properties of two aluminosilicate zeolites, natrolite (NAT) and analcime (ANA), at ambient conditions. An inversion of the acoustic velocity data results in the full set of elastic stiffness moduli $\left(C_{i j}\right.$ 's) for both materials. From the single-crystal moduli the aggregate adiabatic bulk moduli $\left(K_{S}\right)$, shear moduli $(G)$, and Poisson's ratios $(\nu)$ were found to be $K_{s}=48.5(1.0) \mathrm{GPa}, G=31.6(1.0) \mathrm{GPa}$, and $\nu$ $=0.232(5)$ for NAT, and $K_{s}=59.8(1.2) \mathrm{GPa}, G=32.1(1.0) \mathrm{GPa}$, and $\nu=0.272(5)$ for ANA (Voigt-Reuss-Hill averages). The bulk and shear moduli of both zeolites are relatively low compared with those of densely packed aluminosilicates, reflecting an open framework structure of $\left(\mathrm{Al}, \mathrm{SiO}_{4}\right)$ tetrahedra which is easily deformed by bending the $\mathrm{Si}-\mathrm{O}-\mathrm{Al}$ angles. As expected for a less dense crystal, NAT is softer and more compressible than ANA. An evaluation of the directional Young's moduli shows that the compressibility of NAT is nearly uniform along the [100] and [010] axes, while [001] is stiffer, in agreement with previous compression studies. We do not find experimental evidence of negative Poisson's ratios for NAT zeolites as predicted by recent theoretical calculations. (C) 2005 American Institute of Physics. [DOI: 10.1063/1.2014932]
\end{abstract}

\section{INTRODUCTION}

Zeolites are both naturally occurring aluminosilicate minerals and important synthetic materials. They crystallize in a variety of low-density framework nanostructures built from corner-connected $\left(\mathrm{Al}, \mathrm{SiO}_{4}\right)$ tetrahedra which define a narrow size distribution of pores and channels with molecular dimensions. These pores contain charge-balancing cations and molecular water. ${ }^{1}$ The increasing interest surrounding zeolite structures derives from their widespread industrial applications, ${ }^{2}$ such as pollution control, radioactive waste disposal, gas purification, and petroleum production. These applications make use of the unique physicochemical properties of zeolites, including selective ionic exchange, catalysis, and related molecular sieve properties. ${ }^{3}$

For this reason, a number of experimental and theoretical studies have been carried out in recent years to investigate the structural and thermal properties of zeolites. ${ }^{4-8}$ These studies reveal that the unusual framework topology of zeolites allows for "negative" thermal and mechanical behaviors. For example, several zeolite materials are among the few known examples of solids with a negative thermal-

\footnotetext{
a) Author to whom correspondence should be addressed; electronic mail: carmensv@express.cites.uiuc.edu
}

expansion (NTE) coefficient. ${ }^{4,5}$ Recent force-field molecular calculations also predict that a number of zeolite structures possess negative Poisson's ratios, ${ }^{8}$ that is, they expand laterally in tension and contract laterally in compression. Materials exhibiting the later unconventional property, also found in other inorganic compounds as foams, polymers, or the mineral cristobalite, ${ }^{9-11}$ are known as auxetic materials. ${ }^{12}$

Apart from their purely scientific interest, auxetic materials can enhance many material properties (e.g., shear stiffness and indentation resistance) and as a consequence they have numerous potential technological applications. ${ }^{12-14}$ For instance, the auxetic behavior in zeolites could modify their capacity for adsorption, diffusion, or reaction by the application of strain along specific axes. ${ }^{8}$ Due to the scarcity of experimental data on the single-crystal elastic properties of zeolites, ${ }^{15}$ it is difficult to confirm these theoretical predictions.

Brillouin scattering is an accurate nondestructive technique for investigating the elastic properties of small single crystals. It allows direct measurement of the sound velocity along general directions in a transparent medium and hence the determination of the elastic stiffness tensor $C_{i j}$, as well as aggregate properties, including Poisson's ratio. For instance, Brillouin scattering was used to experimentally determine 
that cristobalite ${ }^{11}$ has a negative Poisson's ratio, providing the only experimental evidence of auxetic behavior in a crystalline silicate.

In this paper we report single-crystal measurements on the sound velocities and the elastic properties of natrolite (NAT) and analcime (ANA) zeolites using the Brillouin scattering technique. The acoustic velocities measured at ambient conditions in the samples have been inverted to determine the full set of elastic moduli $C_{i j}$ 's for both materials. These results were used to evaluate the aggregate elastic properties and directional dependence of Young's moduli and Poisson's ratios along the major crystallographic planes.

\section{EXPERIMENT}

\section{A. Sample preparation}

Colorless naturally occurring single crystals of natrolite from County Antrim (Northern Ireland, UK) were used in this study. The structural formula, as determined by energy dispersive $\mathrm{x}$-ray analysis (EDXA), is close to the ideal $\mathrm{Na}_{2}\left(\mathrm{Al}_{2} \mathrm{Si}_{3} \mathrm{O}_{10}\right) \cdot 2 \mathrm{H}_{2} \mathrm{O}$ composition. Natrolite crystallizes in the orthorhombic system, space group $F d d 2,{ }^{16}$ and the lattice parameters determined by single-crystal $x$-ray diffraction are $a=18.350(8) \AA, b=18.622(7) \AA$, and $c=6.600(3) \AA$. The measured composition and the unit-cell volume yield a calculated density of $\rho=2.239(5) \mathrm{g} \mathrm{cm}^{-3}$.

Analcime crystals were synthesized via the bulk-material dissolution technique ${ }^{17,18}$ at the University of Exeter. This technique is designed to produce large $(>1 \mathrm{~mm})$ zeolite crystals. $\mathrm{NaOH}(0.9914$ g, $25 \mathrm{mmol}$, Fisher analytical grade) was dissolved in distilled $\mathrm{H}_{2} \mathrm{O}(6 \mathrm{ml}, 333 \mathrm{mmol})$ in a polytetrafluoroethylene (PTFE)-lined stainless-steel autoclave with a volume of $15 \mathrm{ml}$. Pieces of a ceramic boat $(2.3412 \mathrm{~g}$, Fisher BSH-320-070S) were added as a bulk $\mathrm{SiO}_{2} / \mathrm{Al}_{2} \mathrm{O}_{3}$ source. EDX analyses show that the ceramic boat is mainly $\mathrm{SiO}_{2} / \mathrm{Al}_{2} \mathrm{O}_{3}$ in a $1.4: 1$ ratio. Small amounts of $\mathrm{K}$ $(\sim 1.4$ at. $\%)$ and $\mathrm{Fe}(\sim 0.4$ at. $\%)$ were also observed. After heating at $200{ }^{\circ} \mathrm{C}$ for 2 weeks the autoclave was cooled to room temperature in air and the contents filtered and washed with distilled water. The products consisted of $2.7420 \mathrm{~g}$ of material, including unreacted remains of the ceramic boat and large trapezohedral trisoctahedral crystals of around $2 \mathrm{~mm}$ across. EDX analysis of the crystals gave $1 \mathrm{Al}: 1.15 \mathrm{Na} 2.23 \mathrm{Si}$ (at. \%) with a structural formula of $\mathrm{Na}_{1.05}\left(\mathrm{Al}_{0.95} \mathrm{Si}_{2.0} \mathrm{O}_{6}\right) \cdot \mathrm{H}_{2} \mathrm{O}$. Powder $\mathrm{x}$-ray-diffraction measurements performed on a Bruker D8 Advance diffractometer with $\mathrm{Cu} K \alpha$ radiation confirmed the sample to be analcime with lattice parameter of $a=13.752(5) \AA$ (cubic, space group $I a 3 d) .{ }^{19}$ The calculated density of the sample is $\rho$ $=2.249(4) \mathrm{g} \mathrm{cm}^{-3}$.

The elasticity of orthorhombic natrolite is completely characterized by nine independent single-crystal elastic constants $\left(C_{i j}\right.$ 's). Crystals of high optical quality and free of any inclusion were preoriented using both crystal morphology and $\mathrm{x}$-ray diffraction and polished into plates (150-350 $\left.\times 150 \times 50-30 \mu \mathrm{m}^{2}\right)$ with faces approximately parallel to the (110), (010), and (001) crystallographic planes. These selected orientations allow us to independently and accurately determine the on-diagonal elastic constants $\left(C_{i i}\right)$. Anal- cime is cubic and has only three independent elastic constants $\left(C_{11}, C_{44}\right.$, and $\left.C_{12}\right)$ which can be obtained by measuring the acoustic velocities in a few nonequivalent crystallographic directions in a single crystallographic plane. An optically clear $70-\mu \mathrm{m}$-thick plate with lateral dimensions of $560 \times 350 \mu \mathrm{m}^{2}$ was prepared for the experiments. For Brillouin scattering measurements, the samples were mounted on goniometer heads and their orientation checked using a four-circle single-crystal x-ray diffractometer.

\section{B. Brillouin scattering measurements}

Brilloun scattering measurements were performed using an Ar-ion laser $\left(\lambda_{0}=514.5 \mathrm{~nm}\right)$ as a light source and a $90^{\circ}$ symmetric (platelet) scattering geometry for collection. The scattered light was frequency analyzed with a six-pass FabryPérot interferometer of the Sandercock type, ${ }^{20}$ and detected by a solid-state photon detector. Further details of the experimental setup can be found elsewhere. ${ }^{21}$ In platelet geometry, the measured Brillouin shifts are independent of the refractive index of an optically isotropic material and the acoustic velocities are directly calculated through the relation

$$
V_{i}=\Delta \nu_{i} \lambda_{0} / 2 \sin (\theta / 2),
$$

where $V_{i}$ is the phonon velocity of the compressional $\left(V_{P}\right)$ or shear $\left(V_{S}\right)$ mode, $\Delta \nu_{i}$ the corresponding measured Brillouin frequency shift, $\lambda_{0}$ the laser source wavelength $(514.5 \mathrm{~nm})$, and $\theta$ is the angle between the incident and scattered light $\left(90^{\circ}\right)$. We approximate natrolite $(\delta=0.012)$ as optically isotropic in the data analysis. This assumption is justified by the negligible effect of optical anisotropy on velocity calculation, as demonstrated in the case of Brillouin measurements on highly anisotropic materials, such as carbonate minerals. ${ }^{22}$ The accuracy and reproducibility of our Brillouin system were tested before the experiments using a $\mathrm{MgO}$ single-crystal standard. The reproducibility in repeated measurements was found to be better than $0.5 \%$ of the measured velocity.

Spectra of excellent quality with a high signal-to-noise ratio were recorded with typical exposure times of 5-10 min at an input laser power of $100 \mathrm{~mW}$. All the Brillouin spectra displayed very sharp peaks characteristic of high-quality single crystals (Fig. 1). In order to accurately determine peak positions (Brillouin shift $\Delta \nu_{i}$ ), both Stokes and anti-Stokes contributions in the spectra were fitted assuming Voigt profiles.

\section{RESULTS AND DISCUSSION}

\section{A. Sound velocities and single-crystal elastic constants}

The acoustic velocities were collected for each sample plate over an angular range of $180^{\circ}$ in increments of $15^{\circ}$ or $30^{\circ}$ from both sides of the sample. At least two separate spectra were collected for each direction, varying the collection position on the sample plate. Most of the collected spectra showed the compressional $\left(V_{P}\right)$ and one shear mode $\left(V_{S}\right)$, whereas two shear modes were observed in only a few directions (Figs. 2 and 3). The combined observations from the natrolite crystals resulted in a total of 166 acoustic velocities 

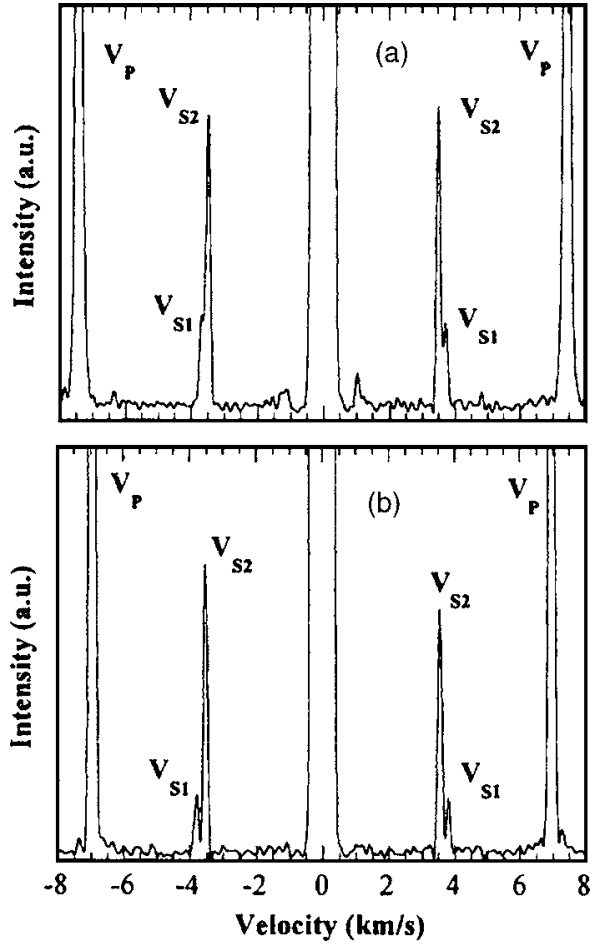

FIG. 1. Representative Brillouin spectra of natrolite (a) and analcime (b) zeolites at ambient conditions. The compressional $\left(V_{P}\right)$ and two shear $\left(V_{S 1}\right.$ and $V_{S 2}$ ) acoustic modes are clearly visible on both the Stokes and antiStokes sides of the spectra. The features at $\pm 1 \mathrm{~km} / \mathrm{s}$ in natrolite spectrum (a) are unattenuated tails of the Rayleigh peak.

(compressional and shear modes) measured in 39 crystallographic directions. For analcime, velocities were collected in 13 distinct crystallographic directions and the final data set consisted of 49 averaged mode velocity measurements.

Phonon velocities are related to the single-crystal elastic moduli through the Christofell's equation, ${ }^{23}$

$$
\operatorname{det}\left|C_{i j k l} n_{j} n_{l}-\rho V^{2} \delta_{i k}\right|=0,
$$

where $C_{i j k l}$ is the elastic modulus tensor, $n_{j}$ and $n_{l}$ the direction cosines of the phonon, $\rho$ the density, $V$ the phonon velocity, and $\delta_{i k}$ the Kronecker delta. The elastic moduli are indicated hereafter by the reduced Voigt notation ${ }^{23} C_{i j}$.

We used a least-square algorithm to calculate the orientations of the phonon directions within a known crystallographic plane from the measured acoustic velocities collected on the sample. The phonon directions, determined with a precision better than $2^{\circ}$, were then used as input data to a linearized inversion procedure ${ }^{24}$ to solve for the independent elastic constants [Eq. (2)]. Inversions were performed with different initial sets of stiffness moduli to verify the independence of the results from the starting model. The best-fit elastic modulus model yields root-mean-square (rms) errors of 0.043 and $0.018 \mathrm{~km} / \mathrm{s}$ with respect to the observed velocities for NAT and ANA samples, respectively. The data sets collected for natrolite and analcime along with the calculated velocity model from the inversion results are shown in Figs. 2 and 3, respectively.

The excellent quality of the least-squares fitting indicates a precision better than $2 \%$ in most of the retrieved elastic constants of both zeolites. The $C_{i j}$ 's are reported in Table I
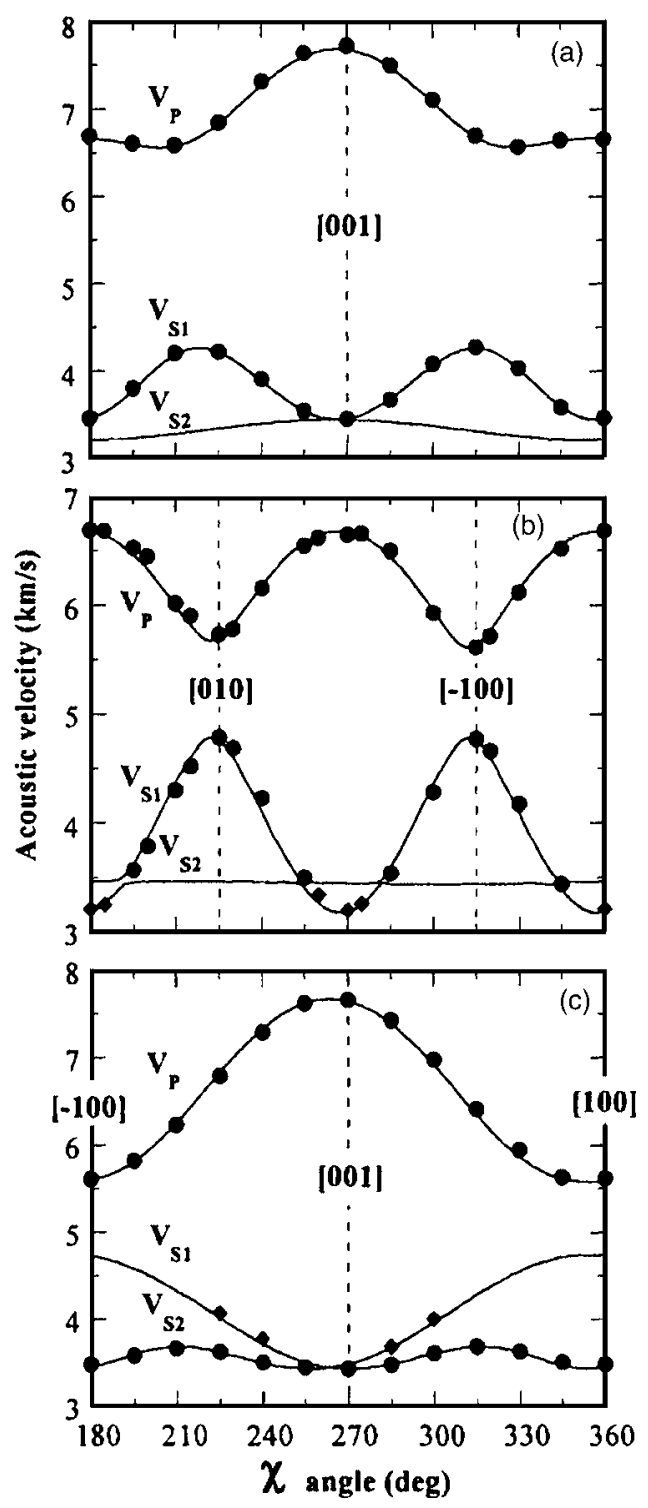

FIG. 2. Measured acoustic velocities in natrolite as a function of crystallographic directions in (a) $(0.7465,0.6654$, and -0.0022$)$, (b) $(-0.0098$, -0.0219 , and 0.9997), and (c) $(-0.0070,0.9950$, and 0.1001) planes. The lines show the acoustic velocities calculated from the best-fit single-crystal elastic moduli. The rms deviation between the calculated and measured velocities is $0.043 \mathrm{~km} / \mathrm{s}$. Uncertainties in the experimental velocities are smaller than the symbol size.

along with previous results for natrolite determined by ultrasonic measurements ${ }^{25}$ and lattice dynamic calculations. ${ }^{26}$ There is no other previous determination of the single-crystal elastic constants of analcime with which our results can be compared. The compliance constants $S_{i j}\left(C_{i j}^{-1}\right)$ obtained in the present study are also reported in Table I.

Comparing the present results with those from previous studies, the difference exceed the estimated error for the present Brillouin measurements $(\sim 1 \%-3 \%)$. The pure compressional $C_{i j}$ constants $\left(C_{11}, C_{22}\right.$, and $\left.C_{33}\right)$ agree with each other to within $10 \%$ whereas larger differences of about $20 \%$ are found in the shear modes (e.g., $C_{44}$ ). The origin of this disagreement is unknown. However, the selected crystallographic planes used in our Brillouin experiments allow us to constraint the on-diagonal compressional and shear 


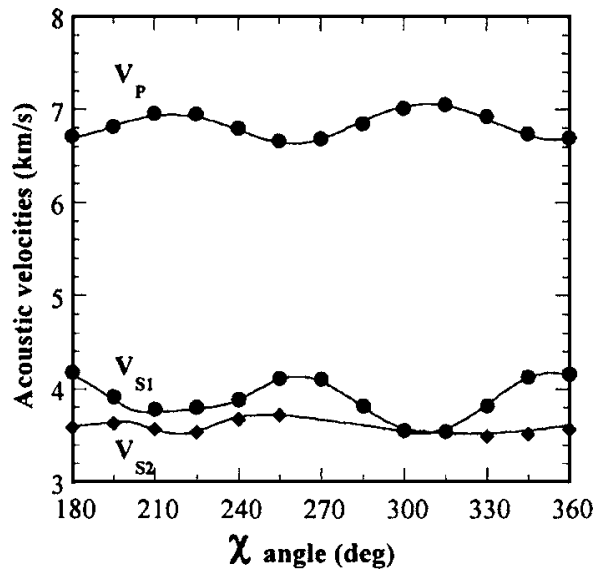

FIG. 3. Measured velocities in analcime as a function of crystallographic directions in the $(-0.9334,0.1146$, and 0.3402$)$ plane. The angle $\chi=360^{\circ}$ corresponds to the $[0.1879,-0.6159$, and 0.7651$]$ direction. The best-fit acoustic velocity model is shown by solid lines. The rms deviation between the calculated and measured velocities is $0.018 \mathrm{~km} / \mathrm{s}$. The error bars on the measured velocities are smaller than the symbol size.

moduli along the principal axes with a high degree of independence and accuracy. Our measurements also provide a high degree of redundancy and cross-checking, ensuring the accuracy of the reported $C_{i j}$ data set.

\section{B. Aggregate properties and elastic anisotropy}

In order to fully characterize the elastic behavior of NAT and ANA zeolites, we have evaluated the aggregate properties and elastic anisotropy. The aggregate adiabatic bulk modulus $\left(K_{s}\right)$, shear modulus $(G)$, and aggregate acoustic velocities were calculated from the $C_{i j}$ 's values using the Voigt-Reuss-Hill (VRH) averaging procedures. The results are reported in Table II together with the aggregate properties calculated from the elastic models found in the literature, ${ }^{25,26}$ using the same averaging method. The bulk modulus of natrolite reported in this work is in excellent agreement with those determined in previous studies ${ }^{25,26}$ (Table II). Our shear modulus is, within the uncertainties, similar to that of Goryainov et al. ${ }^{26}$ but about $13 \%$ higher than the value reported by Ryzhova et $a l^{25}$ The discrepancy is correlated with
TABLE II. Aggregate elastic properties of natrolite and analcime zeolites. All elastic moduli and acoustic velocities, reported as Voigt-Reuss-Hill averages, are given in $\mathrm{GPa}$ and $\mathrm{km} / \mathrm{s}$, respectively.

\begin{tabular}{ccccc}
\hline \hline & \multicolumn{3}{c}{ Natrolite } \\
\cline { 2 - 4 } Parameter & This study & Ref. 25 & Ref. 26 & Analcime \\
\hline$K_{S}$ & $48.5 \pm 1.0$ & 48.9 & 48.3 & $59.8 \pm 1.2$ \\
$G$ & $31.6 \pm 1.0$ & 27.4 & 30.6 & $32.1 \pm 1.0$ \\
$V_{P}$ & $6.36 \pm 0.1$ & 6.17 & 6.31 & $6.75 \pm 0.1$ \\
$V_{S}$ & $3.75 \pm 0.1$ & 3.50 & 3.69 & $3.80 \pm 0.1$ \\
$V_{P} / V_{S}$ & $1.69 \pm 0.03$ & 1.76 & 1.71 & $1.77 \pm 0.03$ \\
$\nu$ & $0.232 \pm 0.005$ & 0.264 & 0.238 & $0.272 \pm 0.005$ \\
$E$ & $77.9 \pm 1.5$ & 69.3 & 75.8 & $81.7 \pm 1.6$ \\
\hline \hline
\end{tabular}

the notable differences in the pure shear constants whereas a better overall agreement is observed in the pure longitudinal elastic constants (Table I).

The isothermal bulk modulus $K_{T}$ of NAT has been obtained in previous measurements of the $P-V$ equation of state by static compression in the diamond-anvil cell (DAC) using x-ray diffraction. ${ }^{27-29}$ Kholdeev et $a l^{27}$ found $K_{T}$ $=47(6) \mathrm{GPa}$ whereas Lee et $a l^{28,29}$ most recently reported a value of 53(1) GPa. Taking into consideration that the isothermal-adiabatic corrections usually amount to about $1 \%$ or less, our present result is in agreement with the value obtained in the compressional data by Kholdeev et $a l .{ }^{27}$ The value of Lee et al. ${ }^{28,29}$ however, differs from our result by $9 \%$, exceeding the mutual uncertainties in the experimental techniques. The discrepancy is unlikely to be explained by compositional differences because both studies utilized natrolite samples close to the ideal $\mathrm{Na}_{2}\left(\mathrm{Al}_{2} \mathrm{Si}_{3} \mathrm{O}_{10}\right) \cdot 2 \mathrm{H}_{2} \mathrm{O}$ composition.

In the work of Lee et al. ${ }^{28,29}$ the reported $K_{T}$ is based only on their data points collected at $P<0.8 \mathrm{GPa}$, presumably before any pressure-induced swelling of the structure due to the selective sorption of water molecules from the fluid pressure-transmitting medium. The discrepancy with our value may in part be due to the difficulty of obtaining a reliable bulk modulus from volume-pressure data over a very limited range of compression. Alternatively, some pressure-

TABLE I. Single-crystal elastic moduli of natrolite and analcime zeolites.

\begin{tabular}{|c|c|c|c|c|c|c|}
\hline \multirow[b]{3}{*}{$i j$} & \multicolumn{4}{|c|}{ Natrolite } & \multicolumn{2}{|c|}{ Analcime } \\
\hline & \multicolumn{3}{|c|}{$C_{i j}(\mathrm{GPa})$} & \multirow{2}{*}{$\begin{array}{c}S_{i j}\left(10^{3} \mathrm{GPa}^{-1}\right) \\
\text { This study }\end{array}$} & \multirow{2}{*}{$\begin{array}{l}C_{i j}(\mathrm{GPa}) \\
\text { This study }\end{array}$} & \multirow{2}{*}{$\begin{array}{c}S_{i j}\left(10^{3} \mathrm{GPa}^{-1}\right) \\
\text { This study }\end{array}$} \\
\hline & This study & Ref. 25 & Ref. 26 & & & \\
\hline 11 & $70.4 \pm 0.7$ & 72.2 & 66.4 & $17.5 \pm 0.3$ & $112.5 \pm 1.1$ & $10.3 \pm 0.2$ \\
\hline 22 & $72.0 \pm 0.7$ & 65.7 & 67.8 & $16.9 \pm 0.3$ & & \\
\hline 33 & $132.3 \pm 1.2$ & 138.0 & 130.7 & $9.0 \pm 0.2$ & & \\
\hline 44 & $26.8 \pm 0.4$ & 19.7 & 33.6 & $37.3 \pm 0.6$ & $27.9 \pm 0.3$ & $35.8 \pm 0.6$ \\
\hline 55 & $26.5 \pm 0.4$ & 24.1 & 34.9 & $37.7 \pm 0.7$ & & \\
\hline 66 & $51.3 \pm 0.6$ & 41.1 & 33.8 & $19.5 \pm 0.4$ & & \\
\hline 12 & $26.0 \pm 0.6$ & 29.6 & 30.1 & $-4.9 \pm 0.1$ & $33.4 \pm 0.4$ & $-2.40 \pm 0.05$ \\
\hline 13 & $32.8 \pm 0.9$ & 25.6 & 32.9 & $-3.20 \pm 0.05$ & & \\
\hline 23 & $31.8 \pm 0.9$ & 36.9 & 31.1 & $-2.80 \pm 0.05$ & & \\
\hline
\end{tabular}


induced absorption of water may have occurred at low pressures but have been difficult to detect via x-ray diffraction.

Concerning analcime, the only available value of $K_{T}$ with which our result can be compared is that published by Hazen and Finger (1979). ${ }^{30}$ These authors measured the isothermal compressibility of ANA up to $3 \mathrm{GPa}$ by singlecrystal X-ray diffraction in the diamond cell, using nonpenetrating pressure-transmitting media. They reported a $K_{T}$ of 40 (1) GPa, which is $30 \%$ lower than the value obtained in our study $\left(K_{s}=59.8 \pm 1.2 \mathrm{GPa}\right)$. The reason for this large discrepancy is uncertain, but conceivably could arise in part from the accuracy of fitting the $P-V$ data to a particular equation of state. An additional source of the discrepancy could be the imprecision in the centering of crystals in DAC experiments, as argued by the authors in a later publication. ${ }^{31}$ In Brillouin measurements, the adiabatic bulk modulus $K_{s}$ is determined independent of any of these factors and can be therefore considered as more accurate than the previous one.

Comparison of the $K_{s}$ values for NAT and ANA zeolites show that NAT is more compressible and more compliant under elongation than ANA. The small bulk and shear moduli of both zeolites (Table II) are related to their relatively open framework structure, which can be easily deformed by bending the $\mathrm{Si}-\mathrm{O}-\mathrm{Al}$ angle involving an oxygen atom shared by the $\left[\mathrm{SiO}_{4}\right]$ and $\left[\mathrm{AlO}_{4}\right]$ tetrahedra. ${ }^{6,32}$ The $K_{s}$ and $G$ values of NAT and ANA are comparable with those reported in acoustic measurements for related tetrahedral frameworks such as $\alpha$-cristobalite ${ }^{11}$ and sodalite ${ }^{33}$ but notably lower than those of dense closed-packed silicates (e.g., stishovite $\left.{ }^{34}\right)$. It is worth noting that the bulk modulus $K_{s}$ of natrolite is smaller than that obtained for the other members of the fibrous group, such as edingtonite ${ }^{35}\left[K_{T}=73(3) \mathrm{GPa}\right]$ or thomsonite ${ }^{35}\left[K_{T}=52(1)\right]$. This distinctive compressional behavior can be attributed either to the smaller pore size in natrolite or to the influence of the type and the amount of extraframework cations located in the structure.

The VRH averaged bulk $\left(K_{s}\right)$ and shear $(G)$ moduli of natrolite and analcime were used to derive aggregate Poisson's ratio $(\nu)$ and Young moduli $(E)$ through the relations (Table II):

$$
\nu=\frac{\left(3 K_{s}-2 G\right)}{\left(6 K_{s}+2 G\right)},
$$

$$
E=2 G(1+\nu)
$$

As seen in Eq. (3), the sign of the Poisson's ratio is determined by the relationship between $K_{s}$ and $G$. In the present study $K_{s}$ is significantly higher than $G$ (Table II), as is typical for a vast majority of crystalline and glassy materials. ${ }^{36}$ The aggregate Poisson's ratios are consequently positive and have values that fall in the common range for most crystalline compounds $(0.20-0.27)$.

The elastic anisotropy of the materials expresses the difference in the stiffness of a structure in different crystallographic directions, and serves as an indicator of their stability. We have computed the anisotropy of natrolite in compressional shear waves and the polarization anisotropy of shear waves in single directions as
TABLE III. Directional Poisson's ratios and Young moduli of natrolite and analcime.

\begin{tabular}{cccc}
\hline \hline Orientaion & Parameter & Natrolite & Analcime \\
\hline (100) plane & $\nu_{23}$ & 0.160 & 0.233 \\
& $\nu_{32}$ & 0.311 & \\
(010) plane & $\nu_{13}$ & 0.183 & 0.233 \\
& $\nu_{31}$ & 0.355 & \\
(001) plane & $\nu_{12}$ & 0.280 & 0.233 \\
& $\nu_{21}$ & 0.290 & \\
{$[100]$ direction } & $E_{1}(\mathrm{GPa})$ & 57.2 & 97.1 \\
{$[010]$ direction } & $E_{2}(\mathrm{GPa})$ & 59.2 & \\
{$[001]$ direction } & $E_{3}(\mathrm{GPa})$ & 111.1 & \\
\hline \hline
\end{tabular}

$$
A_{V_{i}}=\left(V_{i, \max }-V_{i, \min }\right) \times 100 / V_{i, \mathrm{VRH}},
$$

where $\left(V_{i, \text { max }}-V_{i, \min }\right)$ is the difference between the maximum and minimum of the velocities $(i=P$ or $S)$ and $V_{i, \mathrm{VRH}}$ is the corresponding aggregate velocity (Table II).

The present study shows that NAT has a maximum elastic shear anisotropy of $A_{V s}=42 \%$ in the (001) plane, whereas the compressional anisotropy reaches its maximum value $\left(A_{V p}=32 \%\right)$ in the (010) plane [Figs. 2(b) and 2(c)]. The maximum anisotropy for shear waves with orthogonal polarizations is observed close to the [010] and [100] crystallographic axes, and the shear waves velocities differ in these directions by a factor of 1.4 [Fig. 2(b)].

In cubic crystals, the anisotropy is also expressed by an anisotropy factor, ${ }^{37}$ defined as $A=\left(2 C_{44}+C_{12}\right) / C_{11}-1$, which indicates the deviation from elastic isotropy $(A=0)$. We determine an anisotropy factor of $A=-0.207$ for ANA with maximal velocity variations of about $6 \%$ and $15 \%$ for $V_{p}$ and $V_{s}$, respectively (Fig. 3).

\section{Directional Young moduli and Poisson's ratios}

In a single crystal, the magnitude of Young's moduli and Poisson's ratios depends on the specific directions of the applied stress and strain. We have thus evaluated the directional dependence of the Young's moduli as $E_{i}=1 / S_{i i}(i=1,2,3)$, which represents the response to a uniaxial stress applied to the material. The calculated values are listed in Table III and show that the linear compressibility of NAT is nearly the same along the [100] and [010] directions, while the [001] axis is stiffer. This result is in agreement with the static compression studies of Lee et al ${ }^{28,29}$ The fibrous structure of NAT, composed of tetrahedral chains strongly linked along the [001] direction and weakly interconnected in the $x y$ plane, accounts for this compressive behavior.

The directional Poisson's ratio, defined by the quotient of lateral to longitudinal strain for any crystallographic orientation, is given in terms of the single-crystal compliance constants $S_{i j}$ by

$$
\nu_{i j}=-\frac{S_{i j}}{S_{i i}} .
$$

The Poisson's ratios calculated in the major crystallographic planes are listed in Table III. In NAT, the Poisson's ratios exhibit prominent anisotropy, with values ranging from 
+0.355 to +0.160 over the major crystallographic directions NAT and ANA Poisson's ratios are all positives, showing nonauxetic elastic behavior. These results are apparently in contrast with the theoretical predictions of Grima et al., which suggested negative Poisson's ratios for NAT in the (001) plane. These authors reported Poisson's ratios ranging from -0.22 to +0.11 , depending on the force-field model employed in the calculation and on the chemical composition of the sample. The inconsistency between the theory and experiments can be explained by the fact that the simulations were conducted in idealized zeolite cage frameworks, without charge-balancing cations or water molecules in the channels. The presence of extraframework cations in the channels is indeed thought to dramatically change the elastic properties of the zeolites and must be taken into account in the simulation of zeolite structures. The elastic constants for NAT reported in this study may allow refinement of the force-field computational models and, hence, lead to reconciliation between experiments and theoretical calculations.

\section{CONCLUSIONS}

This paper reports on the single-crystal elastic properties of natrolite (NAT) and analcime (ANA) zeolites. The complete set of $C_{i j}$ elastic moduli was accurately determined from the acoustic velocities measured in different crystalline orientations by Brillouin scattering spectroscopy at ambient conditions. The aggregate elastic properties of these materials were evaluated using Voigt-Reuss-Hill (VRH) averaging. This study shows that NAT and ANA framework structures are generally characterized by relatively low bulk $K_{s}$ and shear $G$ moduli, reflecting that they more easily undergo volumetric and shear deformations than many other aluminosilicates.

An evaluation of the directional Young's modulus shows that the compressibility of NAT is nearly uniform along [100] and [010] axes, while [001] is stiffer, in agreement with previous compressive studies. Contrary to recent theoretical calculations, no experimental evidence was found for the predicted auxetic behavior of NAT zeolites. The disagreement, between experiment and theory, can be explained by the fact that efforts to model the zeolite framework did not include cations within the framework structures. The data set of elastic constants for zeolites reported in this study might allow further fine-tuning of the force fields used for molecular mechanics simulations of zeolite structures.

${ }^{1}$ C. Baerlocher, W. M. Maier, and D. H. Olson, Atlas of Zeolite Framework Types (Elsevier, Amsterdam, The Netherlands, 2001).
${ }^{2}$ Handbook of Zeolite Science and Technology, edited by S. M. Auerbach, K. A. Carrrado, and P. K. Dutta (Marcel Dekker, New York, 2003).

${ }^{3}$ D. W. Breck, Zeolite Molecular Sieves (Krieger, Malabar, FL, 1984).

${ }^{4}$ J. W. Couves, R. H. Jones, S. C. Parker, P. Tschaufeser, and C. R. A. Catlow, J. Phys.: Condens. Matter 5, 329 (1993).

${ }^{5}$ D. A. Woodcock, P. Lightfoot, L. A. Villaescusa, M. J. Diaz-Cabanas, M. A. Camblor, and D. Engberg, Chem. Mater. 11, 2508 (1999).

${ }^{6}$ S.-C. Kim, N. R. Keskar, A. V. McCormick, J. R. Chelikowsky, and H. T. Davis, J. Chem. Phys. 102, 8656 (1995).

${ }^{7}$ R. Astala, S. M. Auerbach, and P. A. Monson, J. Phys. Chem. B 108, 9208 (2004)

${ }^{8}$ J. N. Grima, R. Jackson, A. Alderson, and K. E. Evans, Adv. Mater. (Weinheim, Ger.) 12, 1912 (2000).

${ }^{9} \mathrm{R}$. Lakes, Science 235, 1038 (1987).

${ }^{10}$ K. E. Evans, Chem. Ind. 20, 654 (1990).

${ }^{11}$ A. Yaganeh-Haeri, D. Weidner, and J. B. Parise, Science 257, 650 (1992).

${ }^{12}$ K. E. Evans and A. Alderson, Adv. Mater. (Weinheim, Ger.) 12, 617 (2000).

${ }^{13}$ R. H. Baughman, Nature (London) 425, 667 (2003).

${ }^{14}$ W. Yang, Z.-M. Li, W. Shi, B.-H. Xie, and M.-B. Yang, J. Mater. Sci. 39, 3269 (2004).

${ }^{15}$ Z. Wang, R. F. Lobo, and J. Lombros, Microporous Mesoporous Mater. 57, 1 (2003).

${ }^{16}$ G. Artoli, J. V. Smith, and A. Kvick, Acta Crystallogr., Sect. C: Cryst. Struct. Commun. 40, 1658 (1984).

${ }^{17}$ S. Shimizu and H. Hamada, Angew. Chem., Int. Ed. 38, 2725 (1999).

${ }^{18}$ Z. A. D. Lethbridge, J. J. Williams, R. I. Walton, K. E. Evans, and C. W. Smith, Microporous Mesoporous Mater. 79, 339 (2005).

${ }^{19}$ W. H. Taylor, Z. Kristallogr. 113, 430 (1930).

${ }^{20}$ J. R. Sandercock, in Topics in Applied Physics, edited by M. Cardona and G. Güntherodt (Springer, Berlin, 1982), Vol. 51, p. 174

${ }^{21}$ S. V. Sinogeikin, T. Katsura and J. D. Bass, J. Geophys. Res. 103, 20819 (1998).

${ }^{22}$ C.-C. Chen, C.-C. Lin, L.-G. Liu, S. V. Sinogeikin, and J. D. Bass, Am. Mineral. 86, 1525 (2001).

${ }^{23}$ J. F. Nye, Physical Properties of Crystals (Clarendon, Oxford, 1995).

${ }^{24}$ D. J. Weidner and H. R. Carleton, J. Geophys. Res. 82, 1334 (1977).

${ }^{25}$ T. V. Ryzhova, K. S. Aleksandrov, and V. M. Korobkova, Izv., Acad. Sci., USSR, Phys. Solid Earth 2, 63 (1966).

${ }^{26}$ S. V. Goryainov, M. B. Smirnov, and A. P. Shebanin, Dokl. Phys. Chem. 375, 263 (2000)

${ }^{27}$ O. V. Kholdeev, I. A. Belitskii, B. A. Fursenko, and S. V. Goryainov, Dokl. Akad. Nauk SSSR 297, 946 (1987).

${ }^{28}$ Y. Lee, J. A. Hriljac, T. Vogt, J. B. Parise, and G. Artioli, J. Am. Chem. Soc. 123, 12732 (2001).

${ }^{29}$ Y. Lee, J. A. Hriljac, T. Vogt, J. B. Parise, and G. Artioli, J. Am. Chem. Soc. 124, 5466 (2002).

${ }^{30}$ R. M. Hazen and L. W. Finger, Phase Transitions 1, 1 (1979).

${ }^{31}$ R. M. Hazen and L. W. Finger, Am. Mineral. 74, 352 (1989).

${ }^{32}$ M. T. Dove, M. Gambhir, K. D. Hammonds, V. Heine, and A. K. A. Pryde, Phase Transitions 58, 121 (1996).

${ }^{33}$ Z. Li, M. V. Nevitt, and S. Ghose, Appl. Phys. Lett. 55, 1730 (1987).

${ }^{34}$ D. J. Weidner, J. D. Bass, A. E. Ringwood, and W. Sinclair, J. Geophys. Res. 87, 4740 (1982).

${ }^{35}$ Y. Lee, J. A. Hriljac, A. Studer, and T. Vogt, Phys. Chem. Miner. 31, 22 (2004).

${ }^{36}$ J. D. Bass, Mineral Physics and Crystallography. A Handbook of Physical Constants (AGU, Washington, DC, 1995).

${ }^{37}$ B. B. Karki, L. Stixrude, S. J. Clark, M. C. Warren, G. J. Ackland, and J. Crain, Am. Mineral. 82, 51 (1997). 